\title{
The Evaluation of the Set-Up Differences Between Radiation Therapists for Head and Neck Patients
}

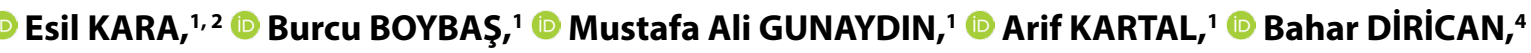 \\ Müge AKMANSU, 3 (D) Ayşe HiçSÖNMEZ ${ }^{1,2}$
}

\author{
'Department of Radiation Oncology, ONKO Ankara Oncology Center, Ankara-Turkey \\ 2Department of Radiation Oncology, Koru Ankara Hospital, Ankara-Turkey \\ ${ }^{3}$ Department of Radiation Oncology, Gazi University, Ankara-Turkey \\ ${ }^{4}$ Department of Radiation Oncology, Gülhane Training and Research Hospital, Ankara-Turkey
}

\begin{abstract}
OBJECTIVE
The aim of the present study was to determine the electronic portal imaging (EPI) evaluation differences between the therapists in the reference of cone beam computed tomography (СBCT).

\section{METHODS}

In the present study, 62 EPI images belonging to 13 head and neck patients were evaluated separately by four therapists as offline, and the amount of shift in the center of fields was determined. CBCT obtained at the same time with the EPI images was accepted as reference, and the amount of shift in the center of fields was compared separately for each therapist with the results of EPI.
\end{abstract}

\section{RESULTS}

According to our results, the amount of shift in the center of fields had changed between therapists with $0-9.4 \mathrm{~mm}$ in the reference of CBCT. The probability of shifting center of fields to be $>3 \mathrm{~mm}$ was $60 \%$ for the first therapist, $35 \%$ for the second therapist, $63 \%$ for the third therapist, and $50 \%$ for the fourth therapist. The probability of shifting center of fields to be $>5 \mathrm{~mm}$ was $24 \%, 8 \%, 27 \%$, and $14.5 \%$, respectively. Analysis of variance for repeated measures test was applied to center shift values, and there were a significant difference in the groups (sig. $<0.05$ ) and a significant difference between the groups (sig. $<0.05$ ).

\section{CONCLUSION}

The usage of CBCT for the verification of treatment fields eliminates the differences in interpersonal evaluation. СBCT improves the set-up accuracy such that planning tumor volume expansion margin can be safely dropped. Therefore, CBCT should be the preferred imaging modality in intensity-modulated radiation therapy planning.

Keywords: CBCT; head and neck; IGRT.

Copyright $\odot$ 2019, Turkish Society for Radiation Oncology

\section{Introduction}

Radiation therapy plays an important role in the management of head and neck cancer (HNC). Cancers arising in the head and neck sites are in close proximity to several critical structures, such as the spinal cord, brainstem, parotid glands, eyes, optic nerves, chiasma, and cochlea. Using intensity-modulated radiation therapy (IMRT) increases the success of treatment while reducing side effects. IMRT has the ability to cover tar- 
get volume and to reduce the doses of the critical organs much greater than conformal radiotherapy. However, there are high-dose gradients between the target volume and critical organs with respect to conformal radiotherapy, and the success of radiotherapy depends on the delivery of the planned doses throughout the entire course of treatment. This can be achieved by the aid of an image-guided radiotherapy (IGRT).

In daily practice, with an increasing number of patients undergoing IGRT, some procedures can give charge to radiation therapists (RTTs). RTTs must be aware of the potential dosimetric impact of position verification procedures, as well as their influence on required margins for HNC radiation therapy. Several studies show that the correctness of the interpretation of RTTs can be improved with proper education and training and may come to a level comparable to radiologists.[1,2]

Studies have shown that using volumetric imaging (cone beam computed tomography (CBCT)) instead of 2D imaging improves the dosimetric results. Li et al. determined that $3 \mathrm{D}$ imaging is more likely to detect shifts $>3 \mathrm{~mm}$ than 2 D imaging (18\% vs. $11 \%)$. [3,4] They found that $\mathrm{CBCT}$ gives more accurate results about translational and rotational errors, whereas $2 \mathrm{D}$ imaging does not give any rotational information. The translational error detected by CBCT is within $0.5 \mathrm{~mm}$, whereas it is $1-2 \mathrm{~mm}$ in $2 \mathrm{D}$ imaging. They found that because of rotational errors, the dose of the spinal cord may increase to $6.4 \%$ and concluded that "small to moderate dosimetric errors" are improved by using CBCT.

The set-up accuracy is important in IMRT to avoid the geographical misses increasing the risk of recurrence. In radiation therapy, the set-up errors in the treatment fields are determined by using portal imaging and $\mathrm{CBCT}$. The images obtained during treatment are compared with the planning images to verify the treatment fields.

In the present study, we aim to determine the electronic portal imaging (EPI) evaluation differences between the therapists in the reference of CBCT.

\section{Materials and Methods}

\section{Patient Set-Up}

Immobilization and CT simulation were performed for 13 patients with HNC, as is routine for patients with HNC receiving IMRT in our department.

Treated patients received their prescribed doses between 10 and 35 fractions. For each patient, megavoltage (MV)-CBCT and electronic portal imaging device (EPID) images were acquired 2 times/week. CBCT was obtained at the same time with the EPI images.

\section{Electronic Portal Imaging}

Before radiotherapy, two orthogonal EPID images (AP or PA and left to right) were acquired using an Optivue 1000ART amorphous silicon flat panel detector. The flat panel has a sensitive area of $409.6 \times 409.6 \mathrm{~mm}$ and an imaging matrix of $1024 \times 1024$ with a resolution of $0.4 \times 0.4 \mathrm{~mm}^{2}$. The images were acquired using coherence therapist software. The bone tissues were marked on the digitally reconstructed radiograph. Thereafter, manual adaptation of the bony anatomy was conducted by RTTs. The set-up error was recorded in two directions in each image, and the error was calculated in three dimensions as shown in Figure 1.

\section{Cone Beam Computed Tomography}

MV-CBCT images were obtained by using the X-ray beam with $4 \mathrm{MV}$ energy produced for only imag-
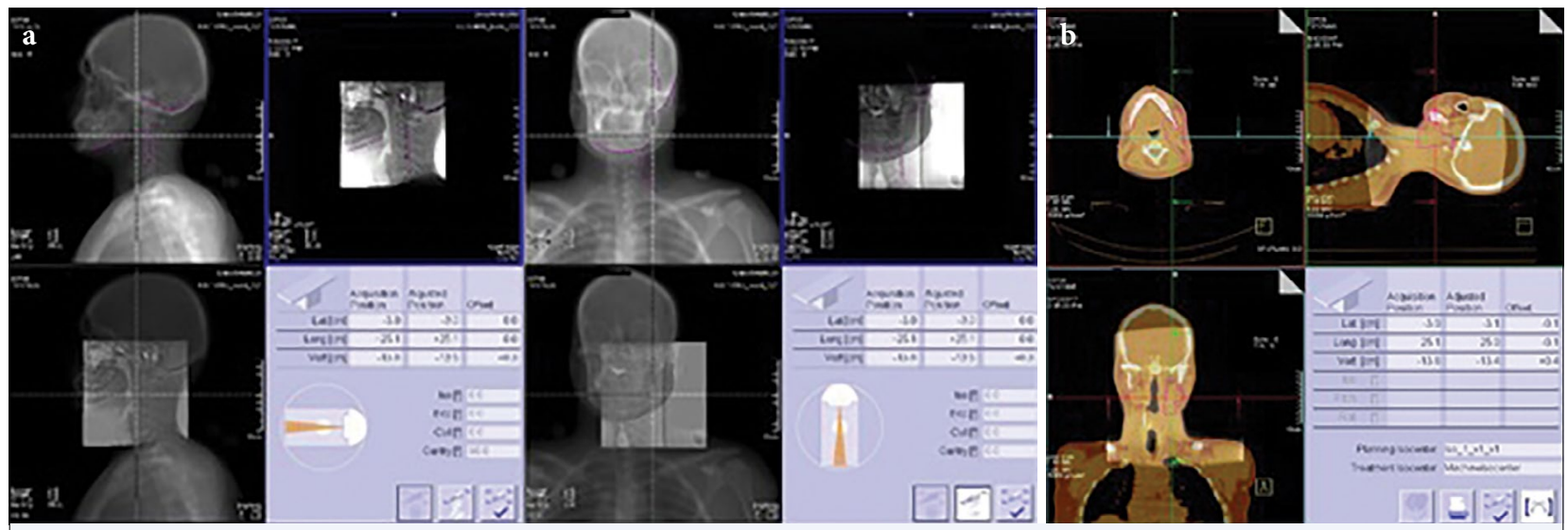

Fig. 1. (a) EPID images of a H\&N patient for Left-Right and AP-PA. (b) Automaticly registered CBCT images. 
ing. The Optivue flat panel detector was attached to the linac on the opposite side of the treatment head at a distance of $1450 \mathrm{~mm}$. Eight MU half circle scan protocol with 200 projections over an arc of 200 was used for obtaining images. By using the flat panel detector, image projections were obtained, and filtered back projection algorithm was used to acquire threedimensional volumetric CT image set. CBCT images were automatically registered to the planning scan using mutual information-based registration algorithm for bone, air, and soft tissue. For checking positional errors, CBCT images were matched with the planning CT. The set-up error was recorded in three directions as shown in Figure 2.

\section{Patient Data}

A total of 62 EPI images belonging to 13 head and neck patients treated in our department were evaluated separately by four therapists as offline, and the amount of shift in the center of fields was determined. CBCT obtained at the same time with the EPI images was accepted as reference, and the amount of shift in the center of fields was compared separately for each therapist with the results of EPI.

The Statistical Package for Social Sciences (SPSS Inc., Chicago, IL, USA) version 22.0 was used for statistical analysis. Analysis of variance for repeated measures (ANOVA) test was applied to center shift values. A $p$ value of $<0.05$ was considered to be significant.

\section{Results}

According to our results, the amount of shift in the center of fields has changed between therapists with 0-9.4 $\mathrm{mm}$ in the reference of CBCT. As can be seen in Figure 3 , there were sometimes even $>5 \mathrm{~mm}$ evaluation differences between the RTT groups.

The probability of shifting center of fields to be $>3$ $\mathrm{mm}$ was $60 \%$ for the first therapist, $35 \%$ for the second therapist, $63 \%$ for the third therapist, and $50 \%$ for the fourth therapist. The probability of shifting center of fields to be $>5 \mathrm{~mm}$ was $24 \%, 8 \%, 27 \%$, and $14.5 \%$, respectively. ANOVA test was applied to center shift values by using the SPSS program, and there were no significant difference in each RTT group (sig. >0.05) (Fig. 4) and a significant difference between the RTT groups (sig. <0.05) (Fig. 5). The degree of impact of the difference between the groups is defined as statistically large.

\section{Discussion}

By using CBCT or 2D imaging systems, the patient is imaged before treatment delivery, and set-up errors could be detected and corrected if necessary. The imaging frequency may be different for each clinic and patient, but it is usually daily. Systematic and random errors can be measured and corrected by this way. In the preparation and implementation of a course of a radiotherapy treatment, at least 17 potential errors are identified.[5] The usage of online corrections can eliminate 14 of these errors. IGRT can provide more

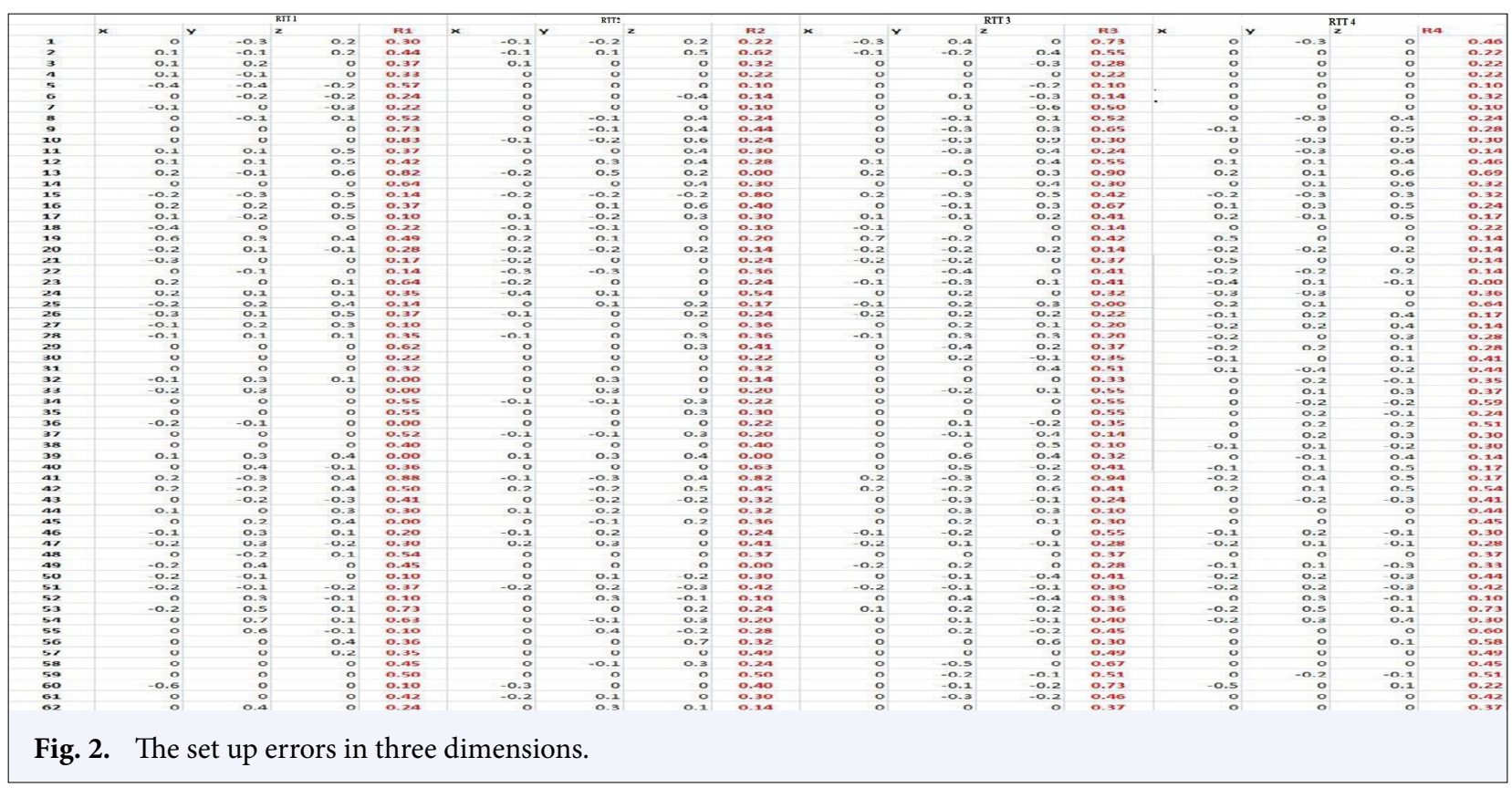




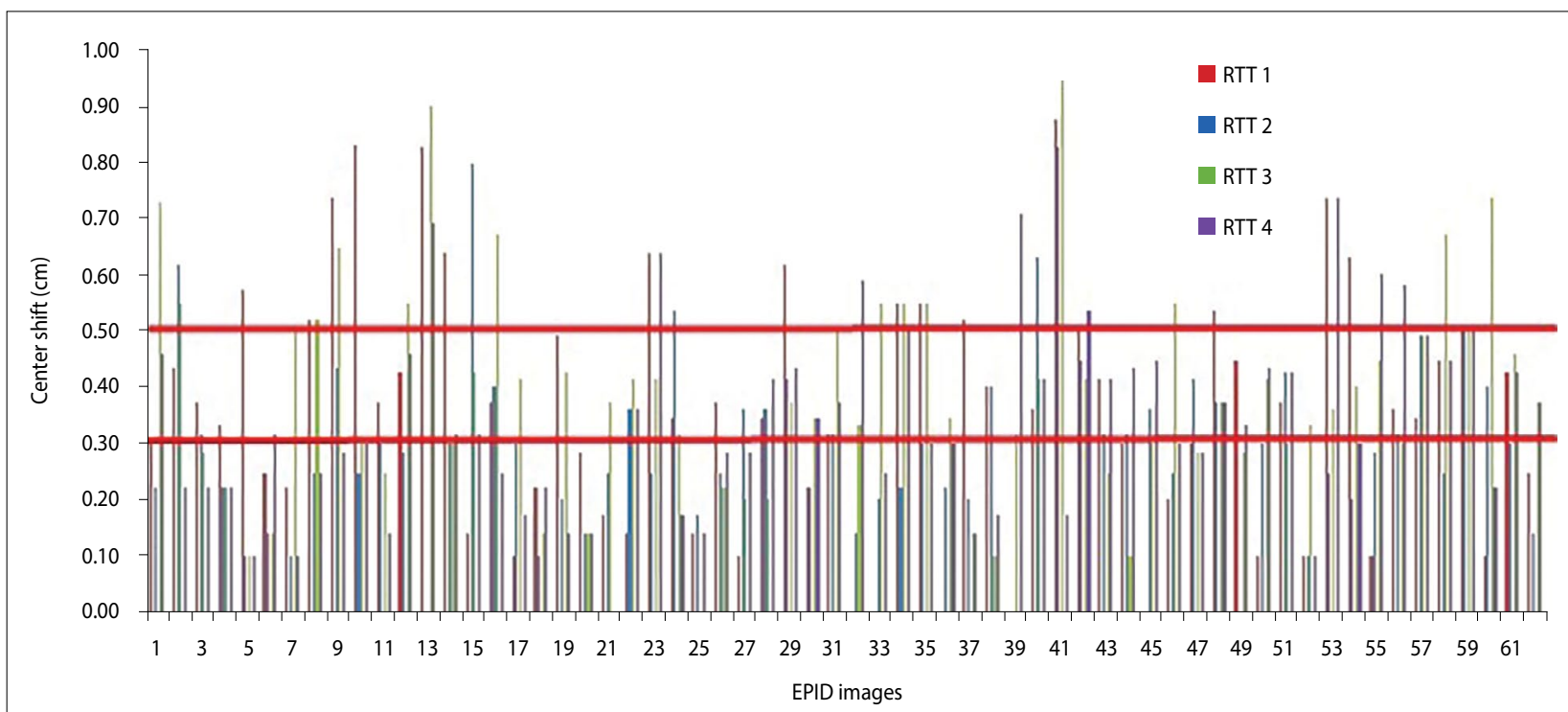

Fig. 3. Amount of shift in the center of fields have changed between RTTs

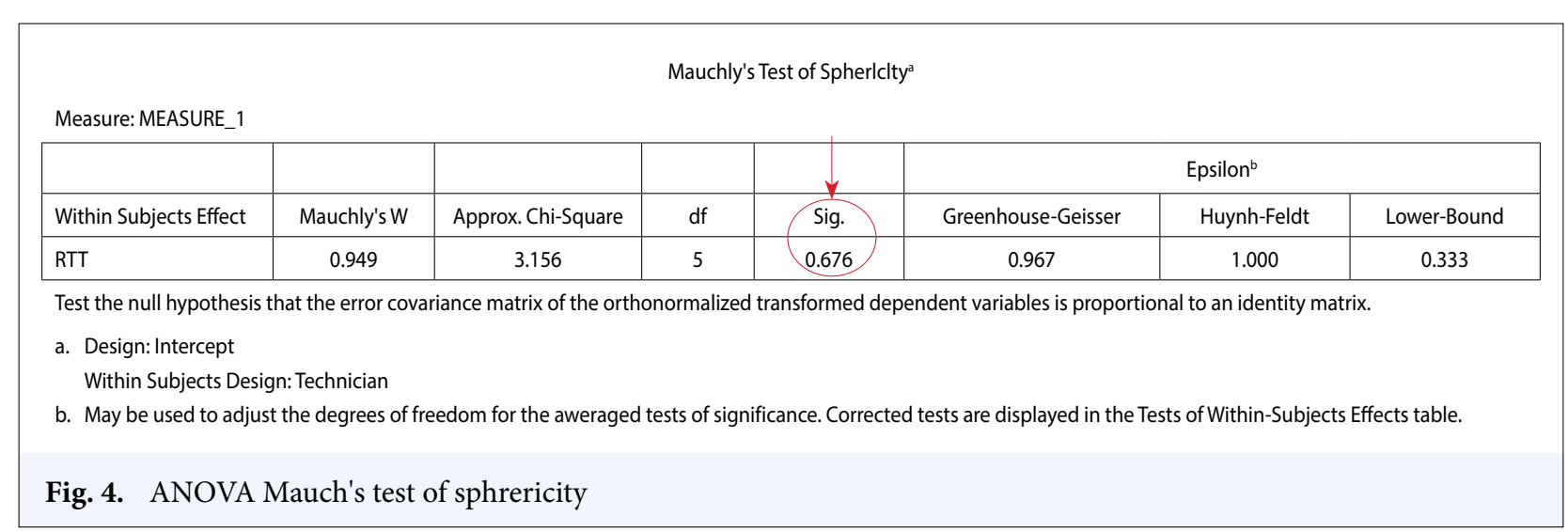

\begin{tabular}{|l|c|c|c|c|c|c|c|}
\hline \multicolumn{7}{|c|}{ Tests of Within-Subjects Effects } \\
\multicolumn{2}{|l|}{ Measure: MEASURE_1 } & Type III Sum of Squares & df & Mean Square & F & Sig. & Partial Eta Squared \\
\hline Source & Sphericity Assumed & 0.270 & 3 & 0.090 & 3.135 & 0.027 & 0.049 \\
\hline RTT & Greenhouse-Geisser & 0.270 & 2.900 & 0.093 & 3.135 & 0.028 & 0.049 \\
& Huynh-Feldt & 0.270 & 3.000 & 0.090 & 3.135 & 0.027 & 0.049 \\
& Lower-Bound & 0.270 & 1.000 & 0.270 & 3.135 & 0.082 & 0.049 \\
\hline \multirow{3}{*}{ Error (Technician) } & Sphericity Assumed & 5.257 & 183 & 0.029 & & & \\
& Greenhouse Geisser & 5.257 & 176.880 & 0.030 & & & \\
& Huynh-Feldt & 5.257 & 183.000 & 0.029 & & & \\
& Lower-Bound & 5.257 & 61.000 & 0.086 & & & \\
\end{tabular}

Fig. 5. ANOVA tests of Within-Subjects Effects.

precise and accurate dose delivery, this tumor will be accurately targeted, the likelihood of tumor control will be increased, and the dose to normal tissues will be reduced.[6]
There are multiple types of technology that can be used for IGRT. The technologies that might be used for $\mathrm{CBCT}$ can include EPIDs, stereoscopic $\mathrm{kV}$ imaging, CBCT, CT-on-rails, MV-CT, X-ray real-time tracking 
systems combining 2D orthogonal $\mathrm{kV}$ and infrared, ultrasound, magnetic resonance imaging, and electromagnetic systems.

The type of system used will depend on the resources of departments and the accuracy of the type of treatments that need to be delivered.

The movements of tumor and tissues may be significant from second to second, day to day, week to week, or longer. The predictable, irregular, or permanent movements may exist in the therapeutic region. Some of them will be significant, whereas others not. Imaging before treatment can overcome movement problems and increase awareness of the range of organ motion, set-up errors, and changes in tumor size and shape that can occur in clinical practice. Keeping patients immobile during treatment, reducing organ movement, and optimizing irradiated volumes provide conformation of the dose around the tumor, achieving greater healthy tissue sparing.

X-ray beams are used to obtain images with good image quality and adequate for detecting bone structures in EPID.[7,8] EPID has many advantages when compared with film-based megavoltage radiography. It offers the adjusting display contrast and assessing target position. In addition, in vivo measurements and three-dimensional dose verification could be done by using EPID.[9] However, it is still difficult to assess field placement with respect to soft tissue structures by using megavoltage radiographs. To reduce the doses of normal tissues, there are requirements for greater accuracy in target localization.

In the present study, set-up differences between RTTs have been evaluated for head and neck patients. We have performed on 13 patients and 62 fractions to determine the evaluation differences of EPI images between therapists. Although the EPID technology has been very much improved, it is still not easy to decide the real location of the bony or soft tissues with EPID. There are many clinics that treat their patients with IMRT technique without using CBCT imaging. However, because of high-dose gradients in IMRT treatments, the set-up accuracy is very important, and the only usage of immobilization materials (e.g., mask and index bar) is not enough to avoid geographical misses. In addition, the evaluation of the EPI images is highly dependent on who evaluates them. The usage of CBCT for the verification of treatment fields eliminates the differences in interpersonal evaluation. CBCT improves the set-up accuracy such that planning tumor volume (PTV) expansion margin can be safely dropped. Therefore, CBCT should be the preferred imaging modality in IMRT planning. Our results suggest that PTV margins can be safely reduced if daily CBCT is possible. Hawkins et al. have also reached the same conclusion in their study.[10] As described by Cheo et al., the usage of daily CBCT allows the PTV expansion to be reduced to as low as $1.2 \mathrm{~mm}$.[11]

\section{Conclusion}

In our study, there is a statistically significant difference between the RTT groups (sig. $<0.05$ ), and the probability of shifting center of fields to be $>3 \mathrm{~mm}$ was $60 \%$ for the first therapist, 35\% for the second therapist, $63 \%$ for the third therapist, and 50\% for the fourth therapist. Using daily CBCT for the verification of radiation field is more convenient with respect to EPID according to our study.

Peer-review: Externally peer-reviewed.

Conflict of Interest: None declared.

Ethics Committee Approval: None declared.

Financial Support: None declared.

Authorship contributions: Concept - E.K., A.H.; Design - E.K.; Supervision - A.H.; Materials - M.A., A.H.; Data collection \&/or processing - B.B., M.A.G., A.K.; Analysis and/ or interpretation - E.K.; Literature search - B.D.; Writing E.K.; Critical review - A.H.

\section{References}

1. Mell LK, Pawlicki T. Image-Guided Radiation Therapy, Perez CA, Halperin EC, Brady LW, editors. Principles and practice of radiation oncology, 5th ed. Philadelphia: Lippincott Williams \& Wilkinspress: 2008. p: 263-93.

2. Şenkesen Ö, Küçücük H. Treatment verification methods. Garipağaoğlu M. Çetingöz R, editors. Basic and Clinical Radiotherapy, first press, Hürriyet printing, TROD publish; 2013.p. 85-95.

3. Smith TN, Baird M. Radiographers' role in radiological reporting: a model to support future demand. Med J Aust 2007;186(12):629-31.

4. Li H, Zhu XR, Zhang L, Dong L, Tung S, Ahamad A, et al. Comparison of $2 \mathrm{D}$ radiographic images and $3 \mathrm{D}$ cone beam computed tomography for positioning head-and-neck radiotherapy patients. Int J Radiat Oncol Biol Phys 2008;71(3):916-25.

5. Kim GY, Pawlicki T, Le QT, Luxton G. Linac-based on-board imaging feasibility and the dosimetric consequences of head roll in head-and-neck IMRT plans. Med Dosim 2008;33(1):93-9. 
6. van Herk M. Errors and margins in radiotherapy. Semin Radiat Oncol. 2004; 14(1):52-64.

7. Michalski J, Purdy JA, Gaspar L, Souhami L, Ballow M, Bradley J, et al. Radiation Therapy Oncology Group. Research Plan 2002-2006. Image-Guided Radiation Therapy Committee. Int J Radiat Oncol Biol Phys 2001;51(3 Suppl 2):60-5.

8. Griffiths SE, Pearcey RG, Thorogood J. Quality control in radiotherapy: the reduction of field placement errors. Int J Radiat Oncol Biol Phys 1987;13(10):1583-8.

9. van Elmpt W, Nijsten S, Mijnheer B, Dekker A, Lam- bin P. The next step in patient-specific QA: 3D dose verification of conformal and intensity-modulated RT based on EPID dosimetry and Monte Carlo dose calculations. Radiother Oncol 2008;86(1):86-92.

10. Boyer AL, Antonuk L, Fenster A, Van Herk M, Meertens $\mathrm{H}$, Munro $\mathrm{P}$, et al. A review of electronic portal imaging devices (EPIDs). Med Phys 1992;19(1):1-16.

11. Hawkins MA, Aitken A, Hansen VN, McNair HA, Tait DM. Set-up errors in radiotherapy for oesophageal cancers--is electronic portal imaging or conebeam more accurate? Radiother Oncol 2011;98(2):249-54. 